\title{
BMJ Open Coronary CT angiography for improved assessment of patients with acute chest pain and low-range positive high- sensitivity troponins: study protocol for a prospective, observational, multicentre study (COURSE trial)
}

\author{
Murat Arslan (1) , , Jeroen Schaap, ${ }^{3}$ Bart Van Gorsel, ${ }^{3}$ Ricardo PJ Budde, ${ }^{1,2}$ \\ Sebastiaan CAM Bekkers, ${ }^{4}$ Yvonne JM Van Cauteren, ${ }^{4}$ Peter Damman, ${ }^{5}$ \\ Jesse Habets, ${ }^{6}$ Eric A Dubois, ${ }^{1,7}$ Admir Dedic ${ }^{1,2}$
}

To cite: Arslan M, Schaap J, Van Gorsel B, et al. Coronary CT angiography for improved assessment of patients with acute chest pain and low-range positive highsensitivity troponins: study protocol for a prospective observational, multicentre study (COURSE trial). BMJ Open 2021;11:e049349. doi:10.1136/ bmjopen-2021-049349

- Prepublication history for this paper is available online. To view these files, please visit the journal online (http://dx.doi org/10.1136/bmjopen-2021049349).

Received 23 January 2021 Accepted 22 September 2021

Check for updates

(c) Author(s) (or their employer(s)) 2021. Re-use permitted under CC BY-NC. No commercial re-use. See rights and permissions. Published by BMJ.

For numbered affiliations see end of article.

Correspondence to

Dr Murat Arslan;

m.arslan@erasmusmc.nl

\section{ABSTRACT}

Introduction Current evaluation of patients suspected of a non-ST-elevation acute coronary syndrome (NSTE-ACS) involves the use of algorithms that incorporate clinical information, electrocardiogram (ECG) and high-sensitivity cardiac troponins (hs-troponins). While primarily designed to rule out NSTE-ACS safely, these algorithms can also be used for rule in of NSTE-ACS in some patients. Still, in a substantial number of patients, these algorithms do not provide a conclusive work-up. These patients often present with an atypical clinical profile and low-range positive hstroponin values without a characteristic rise or fall pattern. They represent a heterogeneous group of patients with various underlying conditions; only a fraction $(30 \%-40 \%)$ will eventually be diagnosed with a myocardial infarction. Uncertainty exists about the optimal diagnostic strategy and their management depends on the clinical perspective of the treating physician ranging from direct discharge to admission for invasive coronary angiography. Coronary CT angiography (CCTA) is a non-invasive test that has been shown to be safe, fast and reliable in the evaluation of coronary artery disease. In this study, we will determine the usefulness of CCTA in patients with acute chest pain and low-range positive hs-troponin values.

Methods and analysis A prospective, double-blind, observational, multicentre study conducted in the Netherlands. Patients aged $30-80$ years presenting to the emergency department with acute chest pain and a suspicion of NSTE-ACS, a normal or non-diagnostic ECG and low-range positive hs-troponins will be scheduled to undergo CCTA. The primary outcome is the diagnostic accuracy of CCTA for the diagnosis of NSTE-ACS at discharge, in terms of sensitivity and negative predictive value.

Ethics and dissemination This study was approved by the Medical Research Ethics Committee of Erasmus Medical Center in Rotterdam, the Netherlands (registration number MEC-2017-506). Written informed consent to participate will be obtained from all participants. This study's findings will be published in a peer-reviewed journal.
Strengths and limitations of this study

- The double-blind nature of this study will enable the assessment of potential policy change(s) that would arise from the use of coronary CT angiography (CCTA) in the emergency department.

- Final diagnosis of non-ST-elevation acute coronary syndrome will be adjudicated by independent cardiologists who are blinded to the results of the CCTA, thereby avoiding the occurrence of incorporation bias.

- This study excludes patients with a history of acute myocardial infarction, percutaneous coronary intervention or coronary artery bypass graft, which has to be taken into account when generalising the results of this study.

Trial registration number ClinicalTrials.gov (NCT03129659).

\section{INTRODUCTION}

Chest pain is the principal symptom of a non-ST-elevation acute coronary syndrome (NSTE-ACS), a broad clinical spectrum ranging from unstable angina pectoris to myocardial infarction (MI). Every year, an estimated 15-20million patients in Europe and the USA present to the emergency department (ED) with chest pain possibly due to an NSTE-ACS and missing this diagnosis can have grave consequences as these patients have higher mortality and morbidity rates if not treated properly. ${ }^{12}$ At present, evaluation involves a clinical examination, ECG and sampling of biomarkers, in the majority of cases of cardiac-specific troponins. Recent years have seen the introduction of new high-sensitivity assays for cardiac troponins, with the capability of detecting smaller amounts of 


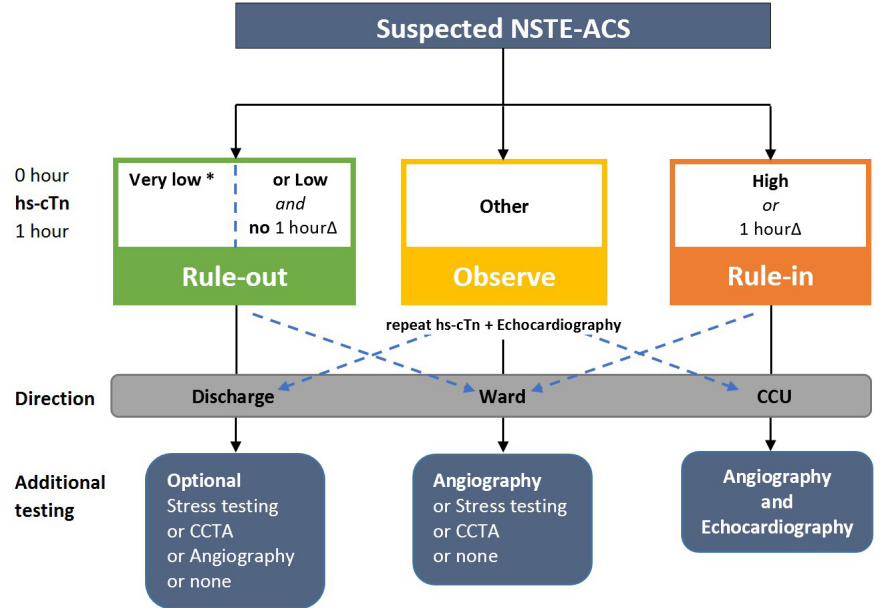

Figure 1 Adopted from the European Society of Cardiology 2020 guidelines. Proposed 0-hour/1-hour algorithm for the diagnostic work-up of suspected non-ST-elevation acute coronary syndrome (NSTE-ACS). *Only applicable if chest pain onset $>3$ hours; $\Delta$, delta. CCTA, coronary CT angiography; CCU, coronary care unit; hs-cTn, highsensitivity cardiac troponin.

myocardial injury. International guidelines recommend specific clinical algorithms for the evaluation of NSTE-ACS incorporating high-sensitivity troponin (hs-troponin) values (figure 1). ${ }^{3}$

However, the current approach does not lead to a conclusive work-up in a substantial number of patients, which fall into the 'indeterminate' category. ${ }^{3}{ }^{4}$ These patients present with low-range positive hs-troponins, without a rise or fall pattern that is characteristic for MI. ${ }^{5}$ They represent a heterogeneous group of patients with various underlying conditions; only a fraction (30\%$40 \%$ ) will eventually be diagnosed with an MI. Knowledge of the coronary status is preferred, because missing an MI has grave consequence. However, performing invasive coronary angiography (ICA) routinely is inefficient and burdensome because of its potential complications. At this moment, it is unclear what the optimal strategy is in these patients. Some will face a prolonged observational period in the hospital and will be exposed to potential risks of medical therapy and invasive testing, other might wrongfully be discharged with a missed NSTE-ACS.

Coronary CT angiography (CCTA) is a non-invasive alternative that accurately detects coronary artery disease and can serve as a gatekeeper for coronary angiography. ${ }^{6-8}$ The aim of this study is to investigate whether CCTA can improve the diagnostic work-up of indeterminate hs-troponin patients suspected of NSTE-ACS.

\section{METHODS AND ANALYSIS}

The Coronary CT Angiography for Improved Assessment of Patients with Acute Chest Pain and Low-Range Positive HighSensitivity troponins (COURSE) Study will be conducted according to the principles of the Declaration of Helsinki (10th version, October 2013) and in accordance with the

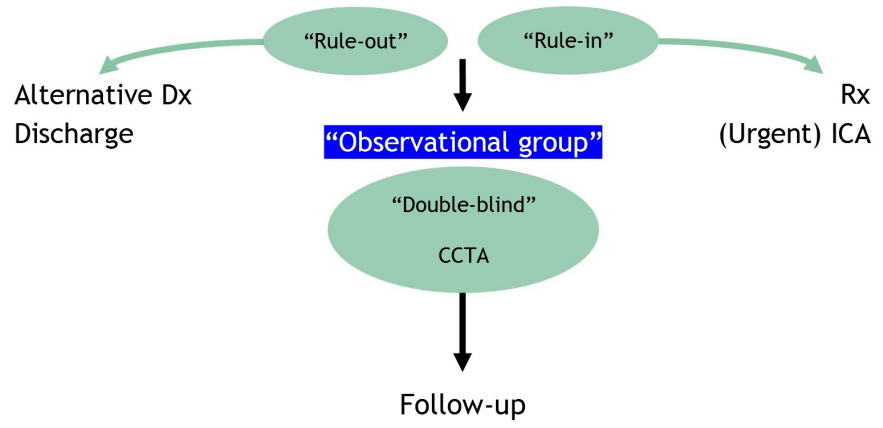

30 days, 6 months,

1 year and 2 year

Figure 2 Study design. Observational group refers to patients who do not meet the criteria for either 'rule-out' or 'rule-in'. CCTA, coronary CT angiography; Dx, diagnosis; ICA, invasive coronary angiography; Rx, medication.

Medical Research Involving Human Subjects Act (WMO). The study is approved by the local institutional review board.

\section{Study design}

This study is a prospective, double-blind, observational, multicentre study. The study began in February 2018 and is currently ongoing. Patients will be recruited at secondary and tertiary care hospitals in the Netherlands. Patients with acute chest pain, a normal or non-diagnostic ECG and low-range positive hs-troponins will be scheduled to undergo CCTA (either at the ED or at the outpatient clinic). The usefulness and potential impact of CCTA on patient management will be evaluated. Patients will be recruited from the emergency ward. Follow-up contacts will take place at 30 days, 6 months, 1 year and 2 years (figure 2).

\section{Inclusion and exclusion criteria}

Patients aged $30-80$ years presenting to the ED with acute chest pain and a suspicion of NSTE-ACS will be eligible. Patients eligible for inclusion are those with low-range positive hs-troponins who do not fulfil criteria for either 'rule-in' or 'rule-out' of NSTE-ACS (figure 3). Exclusion criteria are summarised in box 1.

\section{Recruitment and consent}

Eligible patients presenting to the ED will be informed about the study and asked for informed consent. An independent physician will be available for consultation. Patients willing to participate will provide informed consent in writing.

\section{Patient and public involvement}

Patients or the public were not involved in the design, or conduct, or reporting, or dissemination plans of our research.

\section{CT study protocol}

\section{Coronary calcium scan}

An ECG-triggered CT scan without contrast medium to assess the presence and quantity of calcified coronary plaque. Using the Agatston method, the total amount 


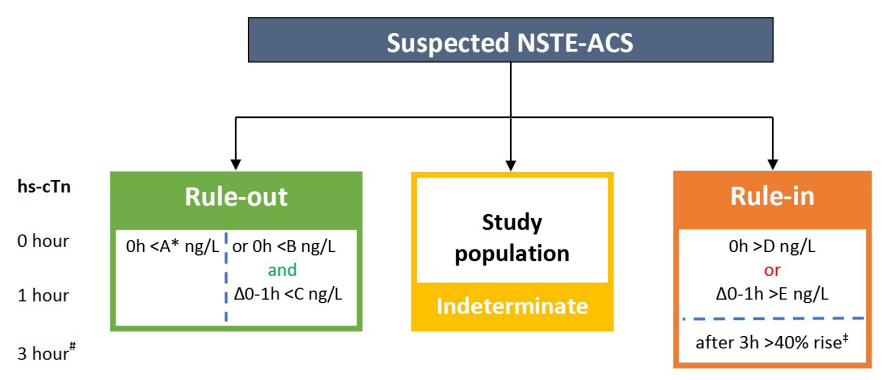

Assay specific cut-off levels in $\mathrm{ng} / \mathrm{L}$ within the $0 \mathrm{~h} / 1 \mathrm{~h}$ algorithms

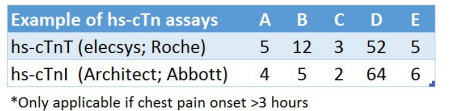

hs-cTnl (Architect; Abbott) $\quad 4 \quad 5 \quad 5 \quad 2 \quad 64 \quad 6$

Only applicable if chest pain onset $>3$ hours

Figure 3 Rule-out and rule-in algorithm, adopted from the European Society of Cardiology 2020 guidelines for the diagnostic work-up of non-ST-elevation acute coronary syndrome (NSTE-ACS). 'O hour/1 hour' rule-out and rulein algorithms using high-sensitivity cardiac troponin (hscTn) assays. \#='0-hour/3-hour' algorithm is only used as a substitute in cases where the standard '0-hour/1-hour' algorithm is not feasible. 0 hour, 1 hour and 3 hour refer to the time (in hours) from first blood draw. hs-cTnl, high-sensitivity cardiac troponin I; hs-cTnT, high-sensitivity cardiac troponin $\mathrm{T} ; \Delta$, delta.

of calcium will be determined using semiautomated software. ${ }^{9}$

\section{Coronary CT angiography}

An ECG-triggered, contrast-enhanced CT scan to image the coronary artery lumen and detect the presence of obstructive coronary disease will be performed. The default scan protocol will be a prospectively ECG-triggered axial CT scan protocol. Retrospective gating may be used with prospectively ECG-triggered tube modulation in patients with an irregular or very fast heart rate. Oral or intravenous metoprolol will be given shortly before the scan for patients with high heart rates, depending on local expertise and CT scanner type, and repeated if necessary. Nitroglycerin sublingual will be given in all patients for

\section{Box 1 Exclusion criteria}

Inability or unwillingness to provide informed consent.

- History of proven CAD, defined as documented prior myocardial infarction, $\mathrm{PCl}$ or CABG surgery.

- Previous examination with either invasive angiography or CCTA in the last 3 years.

- Clinical instability: clinical heart failure, haemodynamic instability, severe chest pain.

- CCTA-specific contraindications: allergy to iodine contrast media; pregnancy; impaired renal function: eGFR $<45 \mathrm{~mL} / \mathrm{min}$; severe arrhythmia likely to affect image interpretation; BMl $>40 \mathrm{~kg} / \mathrm{m}^{2}$; inability to cooperate during the examination.

$\mathrm{BMI}$, body mass index; $\mathrm{CABG}$, coronary artery bypass graft; $\mathrm{CAD}$, coronary artery disease; CCTA, coronary CT angiography; eGFR, estimated glomerular filtering rate; $\mathrm{PCl}$, percutaneous coronary intervention. vasodilation a few minutes before the scan, in the absence of contraindications.

After the scan is performed, the supervising CT reader, at the local hospital, will form a preliminary report. The treating clinician and patient are blinded to the CT results, except in the case of important findings. The results can be classified into the following groups:

1. CCTA reveals findings that do not mandate unblinding of the results.

2. CCTA reveals coronary findings that are shown to have important prognostic implications (significant left main artery disease, significant proximal left anterior descending artery disease, significant three vessel disease). These specific findings will be revealed to the treating physician and patient.

3. CCTA reveals other cardiac (non-coronary) findings that have important prognostic implications, which will be revealed to the treating physician and patient.

4. CCTA reveals significant non-cardiac findings warranting further management or follow-up. Findings will be revealed to the treating physician and patient.

All CT scans which are still blinded to the treating physician at the ED will be systematically read by two experienced $\mathrm{CT}$ readers at the initiating site at a later stage for final reading.

\section{Questionnaires}

Patients will be asked to fill in a questionnaire after 6 months, 1 year and 2 years (or interviewed by telephone when preferred). This is a non-standardised questionnaire to help determine the occurrence of any late major cardiovascular events, medical consumption and current medical therapy. If necessary, patients will be contacted by telephone to clarify their responses at follow-up. With written permission from the participants, information concerning possible medical events will be requested from other healthcare providers if needed.

\section{Outcome measurements}

\section{Primary outcome}

Diagnostic accuracy of $\geq 50 \%$ stenosis on CCTA to identify patients with NSTE-ACS associated with coronary plaque disruption, in terms of sensitivity and negative predictive value.

The diagnosis of NSTE-ACS is established by consensus of two independent cardiologists using all available clinical information including initial clinical presentation, ECG changes, serial laboratory results, (non)-invasive testing and information from the 30-day follow-up. This is considered an acceptable approach considering previous studies with conventional troponins with similar design. ${ }^{10}{ }^{11}$ All medical testing will be performed as indicated by treating physician and is not dependent on study participation. Because of ethical considerations, patients will not undergo standard invasive testing to determine final diagnosis when clinically not indicated. Results of CCTA will not be disclosed to the two independent cardiologists who establish the definitive clinical diagnosis. 


\section{Secondary outcome(s)}

- Diagnostic accuracy of $\geq 70 \%$ stenosis for all coronary segments, except left main ( $\geq 50 \%$ stenosis), on CCTA to identify patients with NSTE-ACS, in terms of sensitivity and negative predictive value.

- The prevalence of atherosclerosis, extent and type, by qualitative analysis.

- Predictive value of CGTA for major adverse cardiac events: death, non-fatal MI and coronary revascularisation at 6 months, 1 year and 2 years.

- Diagnostic accuracy of coronary artery calcium scoring in comparison with CCTA.

- Potential improvement of diagnostic accuracy with fractional flow reserve-computed tomography (FFRCT) in the diagnosis of NSTE-ACS. FFR-CT results will be acquired at a later stage and will not be used for clinical decision-making.

- Subgroup analyses: age, sex, ethnicity and risk scores.

\section{Sample size calculation}

Previous studies show that approximately $40 \%$ of all patients with an inconclusive diagnostic work-up eventually turn out to have NSTE-ACS. ${ }^{5}$ Based on these observations, we assume that the pretest probability of NSTE-ACS will be $40 \%$ in this population. A total of 240 patients are required to demonstrate a negative predictive value of $97 \%$ with a lower margin of $90 \%$, considering an $\alpha=0.05$ and $\beta=0.8$ and a drop-out rate of $10 \%$.

\section{Adverse events}

Adverse events occurring during the CCTA examination include allergic reactions and renal dysfunction. Clinical guidelines apply to avoid complications, and trained personnel are available to handle urgent situations. Although we do not expect the CCTA approach to be associated with an increase in adverse events, we will ask patients to inform us should any unexpected cardiovascular events occur. All adverse events reported spontaneously by the subject or observed by the investigator or his staff will be recorded.

\section{Statistical analysis}

Results will be reported as mean with SD or median with IQRs as appropriate. Data will be presented as categorical or continuous variables, as appropriate. Differences between independent data will be compared using the Student's t-test or the Pearson $\mathrm{X}^{2}$ test, while the paired t-test or the McNemar $\mathrm{X}^{2}$ test will be used for dependent data. Receiver operating characteristic curves with their corresponding areas under the curve (ie, c-statistic) will be constructed to assess the performance of CT. In addition, sensitivity, specificity, negative predictive value and positive predictive value will be calculated with their corresponding $95 \%$ CIs to assess the performance of CT. Variables with $<5 \%$ missing data will be described unchanged, those with 5\%-10\% using imputation methods. In the case of considerable missing data, this variable will not be analysed.

\section{DISCUSSION}

The COURSE trial is a prospective, double-blind, multicentre, observational study to investigate whether CCTA can improve the diagnostic work-up of patients with suspected NSTE-ACS with indeterminate hs-troponins.

The definition of MI has gradually changed with the advent of high-sensitivity cardiac troponin assays. These assays are able to detect very small amounts of myocardial injury or necrosis, with a turnaround time that is much shorter than conventional troponin assays. ${ }^{3}$ In patients with normal and serial low levels of hs-troponins, MI can be ruled out with a negative predictive value that is close to $100 \%$. On one hand, this enables early discharge from the ED of patients with suspected ACS; on the other hand, it allows for expedite detection and treatment of alternative life-threatening causes of acute chest pain. Therapy for an ongoing MI is initiated more swiftly in the case of highly elevated hs-troponins and/or a typical rise or fall pattern during serial sampling, ${ }^{12}$

However, it is estimated that $20 \%-30 \%$ of the patients who present with chest pain suggestive of a cardiac origin do not fulfil the criteria of contemporary algorithms for either rule-in or rule-out of NSTE-ACS. ${ }^{4}$ These patients typically present with a normal or inconclusive ECG and low-range positive hs-troponin values without a typical rise or fall pattern during serial sampling. Elevated hs-troponins indicate myocardial injury, however they do not differentiate the underlying aetiology, which includes atherosclerotic plaque disruption (type $1 \mathrm{MI}$ ), oxygen supply and demand imbalance (type $2 \mathrm{MI}$ ), and nonischaemic myocardial injury. ${ }^{13}$ Differentiating between these conditions may be challenging, but is crucial as they require a different therapeutic approach. Considering that ICA is a costly procedure which carries risks and that eventually less than half of these patients will turn out to have NSTE-ACS, it is sensible to look for more efficient and non-invasive alternatives. ${ }^{5}$

Non-invasive evaluation of coronary artery disease with CCTA CCTA is a well-established diagnostic modality that detects and quantitates coronary artery disease very accurately in a matter of minutes. ${ }^{6-8}$ Currently, it is being employed worldwide as an anatomical alternative to functional testing. ${ }^{14-16}$ Previously, CCTA has successfully been tested in the ED in the assessment of patients suspected of NSTE-ACS. ${ }^{17-19}$ Litt $e t$ $a l$ showed that a CCTA-based strategy in low-risk patients with acute chest pain allowed for a safe and expedited discharge as compared with standard clinical evaluation. ${ }^{18}$ The Rule Out Myocardial Infarction/Ischemia Using Computer Assisted Tomography II trial reinforced these results by showing that early CCTA in low-risk patients with suspected ACS shortened the length of stay in the hospital, while maintaining patient safety. However, this strategy was associated with an increase in downstream testing and did not lead to decrease in cost of care. ${ }^{17}$ It is important to note that these studies were performed in the era of conventional cardiac troponin assays. Since then, hs-troponins have been adopted as the preferred cardiac biomarker and clinical care has changed 
Curved multiplanar reconstructions of the three main coronary artery branches with no signs of coronary artery disease.
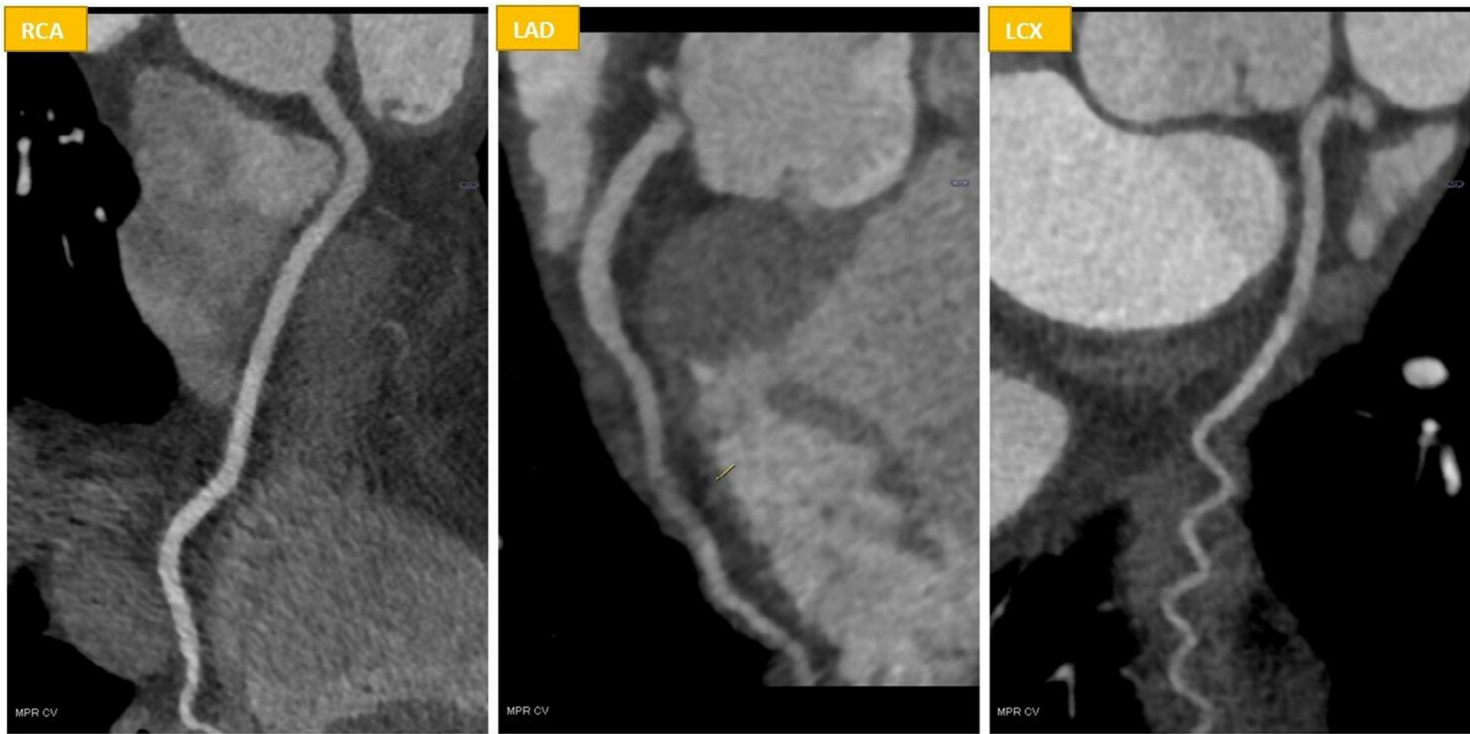

An elderly patient, active smoker, admitted to the ED with typical angina. In-hospital ECG only showed a previously known right bundle branch block. First and second Hs-TnT measurements according to the ESC $0 / 1$ hour algorithm were $13 \mathrm{ng} / \mathrm{L}$ and $11 \mathrm{ng} / \mathrm{L}$, respectively. The patient was assigned to the 'Observe group' and admitted to the Cardiology ward to undergo ICA. By performing CCTA beforehand, which showed no CAD, an unnecessary ICA was avoided.

Figure 4 Exemplary case showcasing the use of CCTA as a gatekeeper for patients with low-range positive high-sensitivity troponin levels in the emergency department (ED). CAD, coronary artery disease; CCTA, coronary CT angiography; ESC, European Society of Cardiology; hs-TnT, high-sensitivity troponin T; ICA, invasive coronary angiography; LAD, left anterior descending artery; LCX, left circumflex artery; RCA, right coronary artery.

substantially. Previously, in the Better Evaluation of Acute Chest Pain with Computed Tomography Angiography trial, we assessed the value of CCTA in the era of hs-troponins for the first time. ${ }^{20}$ Mainly focused on patients with hs-troponins levels beneath the upper reference limit, we found that early CCTA was feasible and resulted in less outpatient testing and lowered direct medical costs. However, it became evident that patients with hs-troponins beneath the upper reference limit are at very low risk of cardiovascular events and might not even require any testing at all. It has been suggested that CCTA might be more beneficial in patients with higher risk. $^{21}$ In patients with low-range positive hs-troponin levels, CCTA might provide a more effective diagnostic work-up. With its excellent negative predictive value, CCTA is an ideal gatekeeper for invasive coronary angiography and might detect other conditions that cause acute chest pain and low-range positive hs-troponin levels (figure 4). Although its diagnostic accuracy slightly declines in patients of older age, CCTA remains a very reliable diagnostic modality. ${ }^{22}$ As an anatomical modality, CCTA does not rely on the provocation of ischaemia, which is unattractive in the case of elevated hs-troponins. Detection of non-obstructive atherosclerotic plaque, considered to be a prerogative for future cardiovascular events and undetected by other diagnostic modalities, provides an opportunity for better prevention measures. ${ }^{23}$ Recently, the Very Early vs Deferred Invasive Evaluation Using Computerized Tomography in Patients With Acute Coronary Syndromes trial, a randomised controlled trial in patients with confirmed NSTE-ACS, showed that CCTA has a high diagnostic accuracy to rule out clinically significant coronary artery disease. In contrast, our observational study will examine whether CCTA can improve the diagnostic work-up of patients presenting with low-range positive hs-troponins, without a rise or fall pattern that is characteristic for MI.

\section{Ethics and dissemination}

This study was reviewed and approved by the Medical Research Ethics Committee of Erasmus Medical Center in Rotterdam, the Netherlands (registration number MEC-2017-506). This study will be conducted according to the principles of the Declaration of Helsinki and in accordance with the Medical Research Involving Human Subjects Act (WMO). Written informed consent to participate will be obtained from all participants. The experimental data will be entered into an electronic case report form. Confidentiality of all participants will be protected by deleting participant identification and privacy information from all study documents. After data storage, only researchers directly involved in the data analysis will be permitted access to the final trial dataset. During the study, local independent data monitoring committees for the various participating centres will be responsible for monitoring the safety, progress, study integrity and design aspects of the trial. This study's findings will be published in scientific peer-reviewed journals and presented at scientific meetings.

\section{Trial status}

Protocol number: version 4-18.07.2019. Recruitment began in February 2018 and is currently ongoing. Expected study completion date: June 2021.

Author affiliations

${ }^{1}$ Cardiology, Erasmus Medical Center, Rotterdam, The Netherlands 
${ }^{2}$ Radiology and Nuclear Medicine, Erasmus Medical Center, Rotterdam, The Netherlands

${ }^{3}$ Cardiology, Amphia Hospital, Breda, The Netherlands

${ }^{4}$ Cardiology, Maastricht University Medical Center, Maastricht, The Netherlands

${ }^{5}$ Cardiology, Radboud University Medical Center, Nijmegen, The Netherlands

${ }^{6}$ Radiology, Radboud University Medical Center, Nijmegen, The Netherlands

${ }^{7}$ Intensive Care, Erasmus Medical Center, Rotterdam, The Netherlands

Contributors $A D$ and EAD conceived and designed the study. EAD obtained funding for the study. MA, $A D$ and EAD drafted the manuscript. MA will coordinate the study and data collection. MA, BVG, and YJMVC are in charge of data acquisition. JS, PD, $\mathrm{SB}, \mathrm{MA}, \mathrm{BVG}, \mathrm{RPJB}, \mathrm{YJMVC}, \mathrm{JH}, \mathrm{EAD}$ and $\mathrm{AD}$ contributed to the writing and review of the manuscript protocol.

Funding This work was supported by a grant from the Erasmus MC Thoraxfoundation (project grant B4).

Disclaimer The funding body has no role in the study design, data collection, data analysis, data interpretation, writing the report or decision to submit the report for publication.

Competing interests None declared.

Patient and public involvement Patients and/or the public were not involved in the design, or conduct, or reporting, or dissemination plans of this research.

Patient consent for publication Not required.

Provenance and peer review Not commissioned; externally peer reviewed.

Open access This is an open access article distributed in accordance with the Creative Commons Attribution Non Commercial (CC BY-NC 4.0) license, which permits others to distribute, remix, adapt, build upon this work non-commercially, and license their derivative works on different terms, provided the original work is properly cited, appropriate credit is given, any changes made indicated, and the use is non-commercial. See: http://creativecommons.org/licenses/by-nc/4.0/.

\section{ORCID iD}

Murat Arslan http://orcid.org/0000-0002-8218-6077

\section{REFERENCES}

1 Pope JH, Aufderheide TP, Ruthazer R, et al. Missed diagnoses of acute cardiac ischemia in the emergency department. $N$ Engl $\mathrm{J}$ Med 2000;342:1163-70

2 Lee TH, Rouan GW, Weisberg MC, et al. Clinical characteristics and natural history of patients with acute myocardial infarction sent home from the emergency room. Am J Cardiol 1987;60:219-24.

3 Collet J-P, Thiele H, Barbato E. The 'Ten Commandments' for the 2020 ESC Guidelines for the management of acute coronary syndromes in patients presenting without persistent ST-segment elevation. Eur Heart J 2020;41:3495-7.

4 Mueller C, Giannitsis E, Christ M, et al. Multicenter evaluation of a 0 -Hour/1-Hour algorithm in the diagnosis of myocardial infarction with high-sensitivity cardiac troponin T. Ann Emerg Med 2016;68:76-87.

5 Nestelberger T, Wildi K, Boeddinghaus J, et al. Characterization of the observe zone of the ESC 2015 high-sensitivity cardiac troponin $0 \mathrm{~h} / 1 \mathrm{~h}$-algorithm for the early diagnosis of acute myocardial infarction. Int J Cardiol 2016;207:238-45.

6 Meijboom WB, Meijs MFL, Schuijf JD, et al. Diagnostic accuracy of 64-slice computed tomography coronary angiography: a prospective, multicenter, multivendor study. J Am Coll Cardiol 2008;52:2135-44
7 Miller JM, Rochitte CE, Dewey M, et al. Diagnostic performance of coronary angiography by 64-row CT. N Engl J Med 2008;359:2324-36.

8 Budoff MJ, Dowe D, Jollis JG, et al. Diagnostic performance of 64-multidetector row coronary computed tomographic angiography for evaluation of coronary artery stenosis in individuals without known coronary artery disease: results from the prospective multicenter accuracy (assessment by coronary computed tomographic angiography of individuals undergoing invasive coronary angiography) trial. J Am Coll Cardiol 2008;52:1724-32.

9 Agatston AS, Janowitz WR, Hildner FJ, et al. Quantification of coronary artery calcium using ultrafast computed tomography. J Am Coll Cardiol 1990;15:827-32.

10 Hoffmann U, Bamberg F, Chae CU, et al. Coronary computed tomography angiography for early triage of patients with acute chest pain: the ROMICAT (rule out myocardial infarction using computer assisted tomography) trial. J Am Coll Cardiol 2009;53:1642-50.

11 Dedic A, Ten Kate G-J, Neefjes LA, et al. Coronary CT angiography outperforms calcium imaging in the triage of acute coronary syndrome. Int J Cardiol 2013;167:1597-602.

12 Reichlin T, Twerenbold R, Wildi $\mathrm{K}$, et al. Prospective validation of a 1-hour algorithm to rule-out and rule-in acute myocardial infarction using a high-sensitivity cardiac troponin T assay. CMAJ 2015;187:E243-52.

13 Thygesen K, Alpert JS, Jaffe AS. Fourth universal definition of myocardial infarction. Eur Heart J 2018.

14 Douglas PS, Hoffmann U. Anatomical versus functional testing for coronary artery disease. N Engl J Med 2015;373:1291-300.

15 SCOT-HEART investigators. CT coronary angiography in patients with suspected angina due to coronary heart disease (SCOTHEART): an open-label, parallel-group, multicentre trial. The Lancet 2015;385:2383-91.

16 Lubbers M, Dedic A, Coenen A, et al. Calcium imaging and selective computed tomography angiography in comparison to functional testing for suspected coronary artery disease: the multicentre, randomized crescent trial. Eur Heart J 2016;37:1232-43.

17 Hoffmann U, Truong QA, Schoenfeld DA, et al. Coronary CT angiography versus standard evaluation in acute chest pain. $N$ Engl J Med 2012;367:299-308.

18 Litt HI, Gatsonis C, Snyder B, et al. CT angiography for safe discharge of patients with possible acute coronary syndromes. $N$ Engl J Med 2012;366:1393-403.

19 Goldstein JA, Chinnaiyan KM, Abidov A, et al. The CT-STAT (coronary computed tomographic angiography for systematic triage of acute chest pain patients to treatment) trial. J Am Coll Cardiol 2011;58:1414-22.

20 Dedic A, Lubbers MM, Schaap J, et al. Coronary CT angiography for suspected ACS in the era of high-sensitivity troponins: randomized multicenter study. J Am Coll Cardiol 2016;67:16-26.

21 Ferencik M, Hoffmann U, Bamberg F, et al. Highly sensitive troponin and coronary computed tomography angiography in the evaluation of suspected acute coronary syndrome in the emergency department. Eur Heart J 2016;37:2397-405.

22 Haase R, Schlattmann P, Gueret P, et al. Diagnosis of obstructive coronary artery disease using computed tomography angiography in patients with stable chest pain depending on clinical probability and in clinically important subgroups: meta-analysis of individual patient data. BMJ 2019;365:I1945.

23 Puchner SB, Liu T, Mayrhofer T, et al. High-risk plaque detected on coronary CT angiography predicts acute coronary syndromes independent of significant stenosis in acute chest pain: results from the ROMICAT-II trial. J Am Coll Cardiol 2014;64:684-92. 